\title{
SOLUTION OF SYMMETRIC POSITIVE SEMIDEFINITE PROCRUSTES PROBLEM*
}

\author{
JINGJING PENG ${ }^{\dagger}$, QINGWEN WANG ${ }^{\dagger}$, ZHENYUN PENG ${ }^{\ddagger}$, AND ZHENCHENG CHEN ${ }^{\S}$
}

\begin{abstract}
In this paper, the symmetric positive semidefinite Procrustes problem is considered. By using matrix inner product and matrix decomposition theory, an explicit expression of the solution is given. Based on the explicit expression given in this paper, it is easy to derive the explicit expression of the solution given by Woodgate [K.G. Woodgate. Least-squares solution of $F=P G$ over positive semidefinite symmetric P. Linear Algebra Appl., 245:171-190, 1996.] and by Liao [A.P. Liao. On the least squares problem of a matrix equation. J. Comput. Math., 17:589-594, 1999.] for the Procrustes problem in some special cases. The explicit expression given in this paper also shows that the solution of the special inverse eigenvalue problem considered by Zhang [L. Zhang. A class of inverse eigenvalue problem for symmetric nonnegative definite matrices. J. Hunan Educational Inst., 2:11-17, 1995 (in Chinese).] can be computed exactly. Examples to illustrate the correctness of the theory results are given.
\end{abstract}

Key words. Symmetric positive semidefinite, Procrustes problem, Matrix inner product, Matrix decomposition.

AMS subject classifications. 15A39, 65F30.

1. Introduction. The symmetric positive semidefinite Procrustes problem:

$$
\min _{P \in S_{\check{L}}^{n}}\|F-P G\|, \quad \text { where } F, G \in R^{n \times m}
$$

is often appeared in many fields such as structural analysis [4], system parameter identification [7], nonlinear programming [12], signal processing [8] and so on.

Procrustes problem (1.1) was first formulated and studied by Allwright [3]. A necessary and sufficient condition for the existence of the minimizer $\widehat{P}$ in (1.1) was given in [12], where the exact global solutions of the Procrustes problem (1.1) are denoted throughout by $\widehat{P}$. The most obvious approach to solve Procrustes problem (1.1) is to write $P=B^{T} B$ and minimize over $B \in R^{n \times n}$. However, as discussed in [11], such a minimization is nonconvex in $B$, and so Procrustes problem (1.1) arise in obtaining a global solution only by using numerical approximation method. How to exactly compute $\widehat{P}$ or, in other words, how to obtain the explicit expression of $\widehat{P}$ is an open problem. Woodgate in [12] given the explicit expression of the solution $\widehat{P}$ for the two special cases, i.e., the case (a): $\widehat{P}=F G^{+}$if $\operatorname{rank}(G)=n$ and $G^{T} F \in S_{\succeq}^{m}$; and the case (b): $\widehat{P}=0$ iff $\operatorname{rank}(G)=n$ and $-F G^{T}-G F^{T} \in S_{\succeq}^{m}$. When $\operatorname{rank}(G)<n$ and assume that the singular value decomposition of the matrix $G$ is

$$
G=U\left(\begin{array}{cc}
\Sigma & 0 \\
0 & 0
\end{array}\right) V^{T}=U_{1} \Sigma V_{1}^{T}
$$

\footnotetext{
* Received by the editors on March 12, 2019. Accepted for publication on August 27, 2019. Handling Editor: Froilan Dopico. Corresponding Author: Peng Zhenyun. Research supported by National Natural Science Foundation of China (11961012), Provincial Natural Science Foundation of Guangxi (2018GXNSFBA281192, 2017GXNSFAA198248), and National Major Scientific Research Instruments Development Program (61627807).

†College of Science, Shanghai University, Shanghai 200444, PR China (jjpeng2012@163.com, wqw@shu.edu.cn).

${ }^{\ddagger}$ School of Mathematics and Computing Science, Guilin University of Electronic Technology, Guilin 541004, PR China (yunzhenp@163.com).

§School of Life and Environments Science, Guilin University of Electronic Technology, Guilin 541004, PR China (chzhch@mail.csu.edu.cn).
} 
where $U=\left(U_{1}, U_{2}\right) \in O^{n \times n}, U_{1} \in R^{n \times r}, V=\left(V_{1}, V_{2}\right) \in O^{m \times m}, V_{1} \in R^{m \times r}, \Sigma=\operatorname{diag}\left(\sigma_{1}, \sigma_{2}, \ldots, \sigma_{r}\right) \succ 0$, $r=\operatorname{rank}(G)$. Then, Liao in [7] given the explicit expression of the solution $\widehat{P}$, under the conditions $\widehat{S} \succeq 0$ and $\operatorname{rank}(\widehat{S})=\operatorname{rank}\left(\left[\begin{array}{ll}\widehat{S} & \widehat{P}_{12}\end{array}\right]\right)$, as

$$
\widehat{P}=U\left(\begin{array}{cc}
\widehat{S} & \widehat{P}_{12} \\
\widehat{P}_{12}^{T} & \widehat{P}_{12}^{T} \widehat{S}^{+} \widehat{P}_{12}+B
\end{array}\right) U^{T},
$$

where $B \in S_{\succeq}^{n-r}$ is arbitrary, $\widehat{S}=\Phi *\left(U_{1}^{T} F V_{1} \Sigma+\Sigma V_{1}^{T} F^{T} U_{1}\right), \widehat{P}_{12}=\Sigma^{-1} V_{1}^{T} F^{T} U_{2}^{T}, \Phi=\left(\frac{1}{\sigma_{i}^{2}+\sigma_{j}^{2}}\right) \in R^{r \times r}$. The authors of the latest literature [5] to consider Procrustes problem (1.1) point out that there is no general procedure for exactly computing the solution $\widehat{P}$ of Procrustes problem (1.1), and so present a semi-analytical approach for the positive semidefinite Procrustes problem (1.1).

Some special cases of Procrustes problem (1.1) have also been studied. For example, a necessary and sufficient condition for solvability and the expression of the solution of the inverse eigenvalue problem

$$
\min _{P \in S}\|G \Lambda-P G\|, \quad \text { where } G \in R^{n \times m} \text { and } \Lambda=\operatorname{diag}\left(\lambda_{1}, \lambda_{2}, \ldots, \lambda_{m}\right)
$$

were given for $S=R^{n \times n}$ and $S=S^{n}$ in $[9,10]$. The special inverse eigenvalue problem

$$
\min _{P \in S_{\succeq}}\|G \Lambda-P G\|, \quad \text { where } G \in R^{n \times m} \text { and } \Lambda=\operatorname{diag}\left(\lambda_{1}, \lambda_{2}, \ldots, \lambda_{m}\right) \succ 0
$$

was studied in [13] by using dual cone theory. However, the author in [13] point out that there is no general procedure for exactly computing the solution of the problem (1.4).

In this paper, we continue study the symmetric positive semidefinite Procrustes problem (1.1). By only using matrix inner product and matrix decomposition theory, the explicit expression of the solutions is derived. Based on the explicit expression given in this paper, it is easy to derive the explicit expression of the solutions, which was given in $[7,12]$, for the symmetric positive semidefinite Procrustes problem (1.1) in some special cases. The explicit expression given in this paper also show that the solution of the problem (1.4) can be computed exactly.

Throughout this paper, $R^{m \times n}, S^{n}, S_{\succeq}^{n}$ and $O^{n \times n}$ denote the set of all $m \times n$ real matrices, $n \times n$ symmetric matrices, $n \times n$ symmetric positive semidefinite matrices and $n \times n$ orthogonal matrices, respectively. $A^{T}, A^{+}$ and $\|A\|$ denote the transpose, Moore-Penrose generalized inverse and the Frobenius norm of the matrix $A$, respectively. $A_{+}$denotes a matrix with $i j$ th entry equals to $\max \left\{0, A_{i j}\right\}$; here $A_{i j}$ denotes the $i j$ th entry of the matrix $A$. The notation $A \succeq B$ means that $A-B$ is a symmetric positive semidefinite matrix and $A \succ B$ means that $A-B$ is a symmetric positive definite matrix. $A * B$ denotes the Hadamard product defined as $A * B=\left(A_{i j} B_{i j}\right)$. $\langle A, B\rangle$ denotes the inner product in matrix space $R^{m \times n}$ defined as $\langle A, B\rangle=\operatorname{trace}\left(B^{T} A\right)=\sum_{i j} A_{i j} B_{i j}$. Obviously, $R^{m \times n}$ is a Hilbert inner product space and the norm of a matrix generated by this inner product space is the Frobenius norm.

2. Solutions for the Procrustes problem (1.1). The necessary and sufficient condition for the existence of the solution of the Procrustes problem (1.1) given in [12] can be described as in the following Lemma 2.1. 
LEMma 2.1. Let

$$
\left(\begin{array}{c}
G_{1} \\
0
\end{array}\right):=U G \quad \text { and } \quad\left(\begin{array}{c}
F_{1} \\
F_{2}
\end{array}\right):=U F
$$

where $U \in O^{n \times n}, G_{1} \in R^{r \times m}, F_{1} \in R^{r \times m}, F_{2} \in R^{(n-r) \times m}$, and rank $\left(G_{1}\right)=r$. Then, the Procrustes problem (1.1) has a solution if and only if

$$
\operatorname{kernel}(\hat{B}) \subseteq \operatorname{kernel}\left(F_{2} G_{1}^{+}\right),
$$

where $\hat{B}$ is the unique solution of the symmetric positive semidefinite Procrustes problem

$$
\min _{B \in S_{\succeq}^{r}}\left\|F_{1}-B G_{1}\right\|^{2} .
$$

By Lemma 2.1, and noting that

$$
\operatorname{kernel}(\hat{B}) \subseteq \operatorname{kernel}\left(F_{2} G_{1}^{+}\right)
$$

if and only if

$$
\operatorname{rank}(\hat{B})=\operatorname{rank}\left(\left[\hat{B}\left(F_{2} G_{1}^{+}\right)^{T}\right]\right),
$$

we can easy obtain the following Lemma 2.2.

Lemma 2.2. Assume that the singular value decomposition of the matrix $G$ is given by (1.2). Then, the symmetric positive semidefinite Procrustes problem (1.1) has a solution if and only if

$$
\operatorname{rank}\left(\hat{P}_{11}\right)=\operatorname{rank}\left(\left[\begin{array}{ll}
\hat{P}_{11} & \Sigma^{-1} V_{1}^{T} F^{T} U_{2}
\end{array}\right]\right),
$$

where $\hat{P}_{11}$ is the unique solution of the symmetric positive semidefinite Procrustes problem

$$
\min _{P_{11} \in S_{\succeq}^{r}}\left\|U_{1}^{T} F V_{1}-P_{11} \Sigma\right\|^{2} .
$$

Why do Woodgate in [12] and Liao in [7] only give the explicit expression of the solution of the Procrustes problem (1.1) in some special cases? Why does the author in [13] point out that there is no general procedure for exactly computing the solution of the problem (1.4)? The mainly reason is that they can not give the explicit expression of the solution of the Procrustes problem (2.1) or (2.6).

In this section, we use matrix inner product and matrix decomposition theory to derive the explicit expression of the solution of the Procrustes problem (2.6), and hence, to derive the explicit expression of the solution of the symmetric positive semidefinite Procrustes problem (1.1). We first introduce to the readers the following Lemmas 2.3-2.5 which are useful to prove the theoretical results in the next content.

Lemma 2.3. ([2]) Assume that

$$
H=\left(\begin{array}{cc}
E & F \\
F^{T} & G
\end{array}\right) \in R^{n \times n}
$$

where $E$ and $G$ are square matrices. Then, $H$ is a symmetric positive semidefinite matrix if and only if

$$
E \succeq 0, G-F^{T} E^{+} F \succeq 0 \quad \text { and } \quad \operatorname{rank}(E)=\operatorname{rank}\left(\left[\begin{array}{ll}
E & F
\end{array}\right]\right) .
$$

Lemma 2.4. ([7]) Assume that the real numbers $\sigma_{1}, \sigma_{2}, \ldots, \sigma_{n}$ are all positive, and $\Phi=\left(\varphi_{i j}\right) \in R^{n \times n}$, $\varphi_{i j}=\frac{1}{\sigma_{i}^{2}+\sigma_{j}^{2}}$. Then, $\Phi \succeq 0$. 
Lemma 2.5. ([6]) If $A, B \in S_{\succeq}^{n}$, then $A * B \in S_{\succeq}^{n}$. If, in addition, $B \in S_{\succ}^{n}$ and $A$ has no diagonal entry equal to 0 , then $A * B \in S_{\succ}^{n}$.

By using matrix inner product theory, we have following Lemma 2.6 and Lemma 2.7.

Lemma 2.6. Assume that $A \in R^{n \times n}$ and $D=\operatorname{diag}\left(d_{1}, d_{2}, \ldots, d_{n}\right) \succ 0$. Then

$$
\langle A D, B D\rangle=0, \quad \forall B \in S^{n}
$$

if and only if $A D^{2}+D^{2} A^{T}=0$.

Proof. If $\langle A D, B D\rangle=0$ for all $B \in S^{n}$, then

$$
\operatorname{trace}\left(D B^{T} A D\right)=\operatorname{trace}(D B A D)=0, \quad \forall B \in S^{n} .
$$

Firstly, let $B=e_{i} e_{i}^{T}$, where $e_{i}(i=1,2, \ldots, n)$ denotes the $i$ th column of the $n \times n$ identity matrix, we have $A_{i i} d_{i}^{2}=0$, i.e., $A_{i i}=0(i=1,2, \ldots, n)$. Then, let $B=\left(e_{i}+e_{j}\right)\left(e_{i}+e_{j}\right)^{T}$. We have by $(2.7)$ and $A_{i i}=0(i=1,2, \ldots, n)$ that $d_{j}^{2} A_{i j}+d_{i}^{2} A_{j i}=0(i, j=1,2, \ldots, n, i<j)$. So, we have $A D^{2}+D^{2} A^{T}=0$.

Conversely, if the matrix $A$ satisfies $A D^{2}+D^{2} A^{T}=0$, we have $d_{i}^{2} A_{i i}=0(i=1,2, \ldots, n)$ and $d_{j}^{2} A_{i j}+d_{i}^{2} A_{j i}=0(i, j=1,2, \ldots, n, i<j)$. Hence, for all $B \in S^{n}$, we have

$$
\langle A D, B D\rangle=\operatorname{trace}(D B A D)=\sum_{1 \leq i \leq n} B_{i i}\left(d_{i}^{2} A_{i i}\right)+\sum_{1 \leq i<j \leq n} B_{i j}\left(d_{i}^{2} A_{i j}+d_{j}^{2} A_{j i}\right)=0 .
$$

Hence, $\langle A D, B D\rangle=0$ holds for all $B \in S^{n}$.

Lemma 2.7. Assume that $F \in R^{n \times n}$ and $D=\operatorname{diag}\left(d_{1}, d_{2}, \ldots, d_{n}\right) \succ 0$. Then there exists the unique $F_{1} \in S^{n}$ and $F_{0} \in\left\{A \in R^{n \times n} \mid A D^{2}+D^{2} A^{T}=0\right\}$ such that

$$
F=F_{1}+F_{0} \quad \text { and } \quad\left\langle F_{1} D, F_{0} D\right\rangle=0 .
$$

Furthermore, $F_{1}$ and $F_{0}$ can expressed as

$$
F_{1}=\Psi *\left(F D^{2}+D^{2} F^{T}\right), \quad F_{0}=\Psi *\left(D^{2} F-D^{2} F^{T}\right),
$$

where $\Psi=\left(\psi_{i j}\right) \in R^{n \times n}, \psi_{i j}=\frac{1}{d_{i}^{2}+d_{j}^{2}}(i, j=1,2, \ldots, n)$.

Proof. Existence. By direct computing, we know that

$$
\begin{gathered}
F_{1}=\Psi *\left(F D^{2}+D^{2} F^{T}\right) \in S^{n}, \\
F_{1}+F_{0}=\Psi *\left(F D^{2}+D^{2} F^{T}\right)+\Psi *\left(D^{2} F-D^{2} F^{T}\right)=F
\end{gathered}
$$

and

$$
F_{0} D^{2}+D^{2} F_{0}^{T}=\left[\Psi *\left(D^{2} F-D^{2} F^{T}\right)\right] D^{2}+D^{2}\left[\Psi *\left(D^{2} F-D^{2} F^{T}\right)\right]^{T}=0,
$$

which means that $F_{0} \in\left\{A \in R^{n \times n} \mid A D^{2}+D^{2} A^{T}=0\right\}$. Hence, we have by Lemma 2.6 that $F_{1}$ and $F_{0}$ given in (2.9) satisfy the conditions (2.8).

Uniqueness. Assume that there also exist $E_{1} \in S^{n}$ and $E_{0} \in\left\{A \in R^{n \times n} \mid A D^{2}+D^{2} A^{T}=0\right\}$ such that $F=E_{1}+E_{0}$ and $\left\langle E_{1} D, E_{0} D\right\rangle=0$. Then $E_{1}-F_{1}=F_{0}-E_{0}$. Hence, we have

$$
\begin{aligned}
& \left\langle\left(E_{1}-F_{1}\right) D,\left(E_{1}-F_{1}\right) D\right\rangle=\left\langle\left(E_{1}-F_{1}\right) D,\left(F_{0}-E_{0}\right) D\right\rangle \\
& =\left\langle E_{1} D, F_{0} D\right\rangle-\left\langle E_{1} D, E_{0} D\right\rangle-\left\langle F_{1} D, F_{0} D\right\rangle+\left\langle F_{1} D, E_{0} D\right\rangle \\
& =\left\langle E_{1} D, F_{0} D\right\rangle+\left\langle F_{1} D, E_{0} D\right\rangle .
\end{aligned}
$$


Since $E_{0}, F_{0} \in\left\{A \in R^{n \times n} \mid A D^{2}+D^{2} A^{T}=0\right\}$, we have

$$
\left\langle E_{1} D, F_{0} D\right\rangle=\left\langle E_{1}, F_{0} D^{2}\right\rangle=\left\langle E_{1},-D^{2} F_{0}^{T}\right\rangle=\left\langle D E_{1},-D F_{0}^{T}\right\rangle=-\left\langle E_{1} D, F_{0} D\right\rangle
$$

and

$$
\left\langle F_{1} D, E_{0} D\right\rangle=\left\langle F_{1}, E_{0} D^{2}\right\rangle=\left\langle F_{1},-D^{2} E_{0}^{T}\right\rangle=\left\langle D F_{1},-D E_{0}^{T}\right\rangle=-\left\langle F_{1} D, E_{0} D\right\rangle,
$$

which mean that $\left\langle E_{1} D, F_{0} D\right\rangle=0$ and $\left\langle F_{1} D, E_{0} D\right\rangle=0$. So, we have $E_{1}=F_{1}$. Analogously, we can prove that $E_{0}=F_{0}$.

Lemma 2.7 implies that any matrix $F \in R^{n \times n}$ can be decomposed into the direct sum of the symmetric matrix set and a special structure matrix set uniquely. In other words, the following Lemma 2.8 holds.

Lemma 2.8. Let $D=\operatorname{diag}\left(d_{1}, d_{2}, \ldots, d_{n}\right) \succ 0$. Then the matrix space $R^{n \times n}$ is a direct sum of the subspace $S^{n}$ and $\left\{A \in R^{n \times n} \mid A D^{2}+D^{2} A^{T}=0\right\}$, i.e.,

$$
R^{n \times n}=S^{n} \oplus\left\{A \in R^{n \times n} \mid A D^{2}+D^{2} A^{T}=0\right\} .
$$

By using Lemma 2.7, it is easy to derive the explicit expression of the symmetric Procrustes problem. This conclusion can be summarized as the following Lemma 2.9 in which the unique symmetric solution is also given by Adhikari and Alam [1] with the different methodologies.

Lemma 2.9. Assume that $F \in R^{n \times n}$ and $D=\operatorname{diag}\left(d_{1}, d_{2}, \ldots, d_{n}\right) \succ 0$. Then the symmetric Procrustes problem

$$
\min _{X \in S^{n}}\|F-X D\|^{2}
$$

is equivalent to the symmetric Procrustes problem

$$
\min _{X \in S^{n}}\left\|\left(F_{1}-X\right) D\right\|^{2},
$$

where $F_{1}=\Psi *\left(F D+D F^{T}\right) \in S^{n}, \Psi=\left(\psi_{i j}\right) \in R^{n \times n}$ and $\psi_{i j}=\frac{1}{d_{i}^{2}+d_{j}^{2}}(i, j=1,2, \ldots, n)$. Furthermore, $\widehat{X}=F_{1}$ is the unique solution of the symmetric Procrustes problem (2.10).

Proof. By Lemma 2.7, there exists the unique $F_{1}=\Psi *\left(F D+D F^{T}\right) \in S^{n}$ and $F_{0}=\Psi *\left(D^{2} F D^{-1}-\right.$ $\left.D F^{T}\right) \in\left\{A \in R^{n \times n} \mid A D^{2}+D^{2} A^{T}=0\right\}$ such that

$$
F D^{-1}=F_{1}+F_{0} \quad \text { and } \quad\left\langle F_{1} D, F_{0} D\right\rangle=0 .
$$

Hence, we have by (2.12) and Lemma 2.6 that

$$
\|F-X D\|^{2}=\left\|\left(F D^{-1}-X\right) D\right\|^{2}=\left\|\left(F_{1}-X\right) D\right\|^{2}+\left\|F_{0} D\right\|^{2}
$$

holds for all $X \in S^{n}$. So, we have

$$
\min _{X \in S^{n}}\|F-X D\|^{2} \Leftrightarrow \min _{X \in S^{n}}\left\|\left(F_{1}-X\right) D\right\|^{2}
$$

Obviously, $\widehat{X}=F_{1}$ is the unique solution of the symmetric Procrustes problem (2.11), and so it is the unique solution of the symmetric Procrustes problem (2.10). 
In order to solve the symmetric positive semidefinite Procrustes problem (1.1), it is necessary introduce to the readers the following Lemma 2.10 and Lemma 2.11.

Lemma 2.10. Assume that $F \in S^{n}$. Then there exists the unique $F_{1}, F_{2} \in S_{\succeq}^{n}$ such that

$$
F=F_{1}-F_{2} \quad \text { and } \quad\left\langle F_{1}, F_{2}\right\rangle=0
$$

Furthermore, if the spectral decompositions of the matrix $F$ is

$$
F=Q\left(\begin{array}{cccc}
\lambda_{1} & & & \\
& \lambda_{2} & & \\
& & \ddots & \\
& & & \lambda_{n}
\end{array}\right) Q^{T}, \quad Q \in O^{n \times n},
$$

then

$$
F_{1}=Q\left(\begin{array}{cccc}
\lambda_{1} & & & \\
& \lambda_{2} & & \\
& & \ddots & \\
& & & \lambda_{n}
\end{array}\right)_{+} Q^{T}, \quad F_{2}=Q\left(\begin{array}{llll}
-\lambda_{1} & & & \\
& -\lambda_{2} & & \\
& & \ddots & \\
& & & -\lambda_{n}
\end{array}\right)_{+} Q^{T} .
$$

Proof. Existence. Obviously, $F_{1}$ and $F_{2}$ given in (2.15) satisfy the conditions (2.13).

Uniqueness. Assume that there also exist $E_{1}, E_{2} \in S_{\succeq}^{n}$ such that

$$
F=E_{1}-E_{2} \quad \text { and } \quad\left\langle E_{1}, E_{2}\right\rangle=0 .
$$

Then $E_{1}-F_{1}=E_{2}-F_{2}$, and so

$$
\begin{aligned}
0 & \leq\left\langle E_{1}-F_{1}, E_{1}-F_{1}\right\rangle=\left\langle E_{1}-F_{1}, E_{2}-F_{2}\right\rangle \\
& =\left\langle E_{1}, E_{2}\right\rangle-\left\langle E_{1}, F_{2}\right\rangle-\left\langle F_{1}, E_{2}\right\rangle+\left\langle F_{1}, F_{2}\right\rangle \\
& =-\left\langle E_{1}, F_{2}\right\rangle-\left\langle F_{1}, E_{2}\right\rangle .
\end{aligned}
$$

Since $E_{1}, E_{2}, F_{1}, F_{2} \in S_{\succeq}^{n}$, we have $\left\langle E_{1}, F_{2}\right\rangle=\operatorname{trace}\left(F_{2}^{T} E_{1}\right) \geq 0$ and $\left\langle F_{1}, E_{2}\right\rangle=\operatorname{trace}\left(E_{2}^{T} F_{1}\right) \geq 0$. So, we have $E_{1}=F_{1}$. Analogously, we can prove that $E_{2}=F_{2}$.

Lemma 2.11. Assume that $F \in R^{n \times n}$ and $D=\operatorname{diag}\left(d_{1}, d_{2}, \ldots, d_{n}\right) \succ 0$, and assume that the spectral decompositions of the matrix $\Psi *\left(F D+F^{T} D\right)$ is

$$
\Psi *\left(F D+D F^{T}\right)=W\left(\begin{array}{cccc}
\gamma_{1} & & & \\
& \gamma_{2} & & \\
& & \ddots & \\
& & & \gamma_{n}
\end{array}\right) W^{T}
$$

where $W \in O^{n \times n}, \Psi=\left(\psi_{i j}\right) \in R^{n \times n}$ and $\psi_{i j}=\frac{1}{d_{i}^{2}+d_{j}^{2}}(i, j=1,2, \ldots, n)$. Then the following symmetric positive semidefinite Procrustes problem

$$
\min _{X \in S_{\succeq}^{n}}\|F-X D\|^{2}
$$


has the unique solution $\widehat{X}$ which can be expressed as

$$
\widehat{X}=W\left(\begin{array}{cccc}
\gamma_{1} & & & \\
& \gamma_{2} & & \\
& & \ddots & \\
& & & \gamma_{n}
\end{array}\right)_{+} W^{T} .
$$

Proof. Let $F_{1}=\Psi *\left(F D+F^{T} D\right)$, we have by Lemma 2.9 that the symmetric positive semidefinite Procrustes problem (2.17) is equivalent to the symmetric positive semidefinite Procrustes problem

$$
\min _{X \in S_{\unrhd}^{n}}\left\|\left(F_{1}-X\right) D\right\|^{2}
$$

By Lemma 2.10, there exists the unique

$$
\bar{F}_{1}=W\left(\begin{array}{cccc}
\gamma_{1} & & & \\
& \gamma_{2} & & \\
& & \ddots & \\
& & & \gamma_{n}
\end{array}\right)_{+} W^{T}
$$

and

$$
\bar{F}_{2}=W\left(\begin{array}{cccc}
-\gamma_{1} & & & \\
& -\gamma_{2} & & \\
& & \ddots & \\
& & & -\gamma_{n}
\end{array}\right)_{+} W^{T}
$$

such that

$$
F_{1}=\bar{F}_{1}-\bar{F}_{2} \quad \text { and } \quad\left\langle\bar{F}_{1}, \bar{F}_{2}\right\rangle=0 .
$$

Hence, we have

$$
\begin{aligned}
\left\|\left(F_{1}-X\right) D\right\|^{2} & =\left\|\left(\bar{F}_{1}-X\right) D\right\|^{2}-2\left\langle\left(\bar{F}_{1}-X\right) D, \bar{F}_{2} D\right\rangle+\left\|\bar{F}_{2} D\right\|^{2} \\
& =\left\|\left(\bar{F}_{1}-X\right) D\right\|^{2}+2 \operatorname{trace}\left(D \bar{F}_{2}^{T} X D\right)+\left\|\bar{F}_{2} D\right\|^{2}
\end{aligned}
$$

holds for all $X \in S_{\succeq}^{n}$. From (2.20) and noting that $D$ is a positive definite diagonal matrix, we know that $\widehat{X}=\bar{F}_{1}$ is the unique solution of the symmetric positive semidefinite Procrustes problem (2.19). Hence, the symmetric positive semidefinite Procrustes problem (2.17) has the unique solution $\widehat{X}$ which can be expression as (2.18).

For the symmetric positive semidefinite Procrustes problem (1.1), we have the following Theorem 2.12.

THEOREM 2.12. Assume that the singular value decomposition of the matrix $G$ is given by (1.2), and assume that the spectral decompositions of the matrix $\widehat{S}=\Phi *\left(U_{1}^{T} F V_{1} \Sigma+\Sigma V_{1}^{T} F^{T} U_{1}\right)$ is

$$
\widehat{S}=\Phi *\left(U_{1}^{T} F V_{1} \Sigma+\Sigma V_{1}^{T} F^{T} U_{1}\right)=N\left(\begin{array}{cccc}
\delta_{1} & & & \\
& \delta_{2} & & \\
& & \ddots & \\
& & & \delta_{r}
\end{array}\right) N^{T},
$$


where $N \in O^{r \times r}, \Phi=\left(\varphi_{i j}\right) \in R^{n \times n}$ and $\varphi_{i j}=\frac{1}{\sigma_{i}^{2}+\sigma_{j}^{2}}(i, j=1,2, \ldots, r)$.

Let

$$
\widehat{P}_{11}=N\left(\begin{array}{cccc}
\delta_{1} & & & \\
& \delta_{2} & & \\
& & \ddots & \\
& & & \delta_{r}
\end{array}\right)_{+}^{N^{T}}
$$

Then the symmetric positive semidefinite Procrustes problem (1.1) has a solution if and only if

$$
\operatorname{rank}\left(\widehat{P}_{11}\right)=\operatorname{rank}\left(\left[\begin{array}{ll}
\widehat{P}_{11} & \widehat{P}_{12}
\end{array}\right]\right)
$$

where $\widehat{P}_{12}=\Sigma^{-1} V_{1}^{T} F^{T} U_{2}$.

Furthermore, if the symmetric positive semidefinite Procrustes problem (1.1) has a solution, then the explicit expression of the solution is

$$
\widehat{P}=U\left(\begin{array}{cc}
\widehat{P}_{11} & \widehat{P}_{12} \\
\widehat{P}_{12}^{T} & \widehat{P}_{12}^{T} \widehat{P}_{11}^{+} \widehat{P}_{12}+P_{22}
\end{array}\right) U^{T}
$$

where $P_{22} \in S_{\succeq}^{n}$ is arbitrary.

Proof. Using the invariance of the Frobenius norm under orthogonal transformations, we have

$$
\|F-P G\|^{2}=\left\|F-P U\left(\begin{array}{cc}
\Sigma & 0 \\
0 & 0
\end{array}\right) V^{T}\right\|^{2}=\left\|U^{T} F V-U^{T} P U\left(\begin{array}{cc}
\Sigma & 0 \\
0 & 0
\end{array}\right)\right\|^{2} .
$$

Let

$$
U^{T} P U=\left(\begin{array}{cc}
P_{11} & P_{12} \\
P_{12}^{T} & P_{22}
\end{array}\right), \quad P_{11} \in R^{r \times r}, \quad P_{22} \in R^{(n-r) \times(n-r)}
$$

Then, we have

$$
\|F-P G\|^{2}=\left\|U_{1}^{T} F V_{1}-P_{11} \Sigma\right\|^{2}+\mid U_{2}^{T} F V_{1}-P_{12}^{T} \Sigma\left\|^{2}+\right\| F V_{2} \|^{2} .
$$

So, the symmetric positive semidefinite Procrustes problem (1.1) is equivalent to

$$
\underset{\substack{P_{11} \in S^{r}, P_{12} \in R^{r \times(n-r)}, \operatorname{rank}\left(P_{11}\right)=\operatorname{rank}\left(\left[P_{11} P_{12}\right]\right)}}{\min }\left\|U_{1}^{T} F V_{1}-P_{11} \Sigma\right\|^{2}+\left\|U_{2}^{T} F V_{1}-P_{12}^{T} \Sigma\right\|^{2} .
$$

By Lemma 2.2, the problem (2.26) has a solution if and only if

$$
\begin{gathered}
\widehat{P}_{11}=\arg \min _{P_{11} \in S_{\succeq}^{r}}\left\|U_{1}^{T} F V_{1}-P_{11} \Sigma\right\|^{2}, \\
\widehat{P}_{12}=\arg \min _{P_{12} \in R^{r \times(n-r)}}\left\|U_{2}^{T} F V_{1}-P_{12}^{T} \Sigma\right\|^{2}=\Sigma^{-1} V_{1}^{T} F^{T} U_{2}
\end{gathered}
$$

and

$$
\operatorname{rank}\left(\widehat{P}_{11}\right)=\operatorname{rank}\left(\left[\begin{array}{ll}
\widehat{P}_{11} & \widehat{P}_{12}
\end{array}\right]\right) .
$$

It follows from Lemma 2.11 that $\widehat{P}_{11}$ is unique and can be expressed as (2.22). Hence, if the symmetric positive semidefinite Procrustes problem (1.1) has a solution, we have by Lemma 2.3 that the solution $\widehat{P}$ can be expressed as (2.24). 
Based on the above results, we have the following Remarks 2.13-2.15.

REMARK 2.13. If $\operatorname{rank}(G)=n$, then $\widehat{P}=U \widehat{P}_{11} U^{T}$. Also, if $G^{T} F \in S_{\succeq}^{m}$, then

$$
F G^{+}=\left(G G^{T}\right)^{-1} G\left(G^{T} F\right) G^{T}\left(G G^{T}\right)^{-1} \succeq 0 .
$$

In addition, it follows from the proof of Lemma 2.9 and the condition $G^{T} F \in S_{\succeq}^{m}$ that

$$
\widehat{S}=\Phi *\left(U^{T} F V_{1} \Sigma+\Sigma V_{1}^{T} F^{T} U\right)=U^{T} F V_{1} \Sigma^{-1}=U^{T} F G^{+} U \succeq 0
$$

So,

$$
\widehat{P}=U \widehat{P}_{11} U^{T}=U \widehat{S}_{11} U^{T}=F G^{+}
$$

If $-F G^{T}-G F^{T} \in S_{\succeq}^{m}$, then, by Lemma 2.4 and Lemma 2.5, $\widehat{S}=\Phi *\left(U^{T}\left(F G^{T}+G F^{T}\right) U\right) \in-S_{\succeq}^{n}$. Hence, $\widehat{P}_{11}=0$, and so $\widehat{P}=0$. These results implies that Theorem 2.12 is a generalization of the results given in $[12]$.

REMARK 2.14. If $\operatorname{rank}(G)<n$ and $\widehat{S}=\Phi *\left(U_{1}^{T} F V_{1} \Sigma+\Sigma V_{1}^{T} F^{T} U_{1}\right) \succeq 0$, then $\widehat{P}_{11}=\widehat{S}$. Hence, Theorem 2.12 is a generalization of the Theorem 3.2 in [7].

REMARK 2.15. Theorem 2.12 implies that the explicit expression of the solution $\widehat{P}$ of the special inverse eigenvalue problem considered in [13] can be computed exactly. This illustrates that the open problems point out in [13] and in [5] can be solved.

3. Algorithm and numerical examples. According to discussion in section 2, we can give an algorithm to solve the symmetric positive semidefinite Procrustes problem (1.1) as following steps:

Step 1. Input matrices $F, G \in R^{n \times m}$.

Step 2. According to (1.2) decomposing the matrix $G$.

Step 3. According to (2.21) decomposing the matrix $\widehat{S}=\Phi *\left(U_{1}^{T} F V_{1} \Sigma+\Sigma V_{1}^{T} F^{T} U_{1}\right)$.

Step 4. According to $(2.22)$ computing $\widehat{P}_{11}$.

Step 5. Computing $\widehat{P}_{12}=\Sigma^{-1} V_{1}^{T} F^{T} U_{2}$.

Step 6. If $\operatorname{rank}\left(\widehat{P}_{11}\right)=\operatorname{rank}\left(\left[\begin{array}{ll}\widehat{P}_{11} & \widehat{P}_{12}\end{array}\right]\right)$, then the symmetric positive semidefinite Procrustes problem (1.1) has a solution. Otherwise, the symmetric positive semidefinite Procrustes problem (1.1) has no solution.

Step \%. According to (2.24) computing $\widehat{P}$, where $P_{22} \in S_{\succeq}^{r}$ is chosen as arbitrary.

In the following number examples, $P_{22}$ (1f it is necessary) is chosen as zero matrix.

ExAmple 3.1. Consider matrices $F$ and $G$ as follows:

$$
F=\left(\begin{array}{rrrrrrr}
6.3043 & -6.5364 & 1.2659 & -2.7625 & -2.9861 & -5.4362 & 2.7422 \\
-0.5694 & -9.4371 & -5.5455 & -15.6041 & 24.4958 & -20.4567 & -11.4576 \\
-0.1030 & 2.3164 & 3.0813 & 8.1280 & -10.6447 & 6.6903 & 7.9874 \\
8.1678 & -4.5977 & 0.0559 & -2.6948 & 1.1326 & -6.5904 & 2.0167 \\
6.3043 & -6.5364 & 1.2659 & -2.7625 & -2.9861 & -5.4362 & 2.7422 \\
-0.5694 & -9.4371 & -5.5455 & -15.6041 & 24.4958 & -20.4567 & -11.4576
\end{array}\right),
$$




$$
G=\left(\begin{array}{rrrrrrr}
0.3452 & -0.9897 & 0.8082 & -0.1739 & -1.4692 & -0.2531 & 1.0339 \\
0.2472 & -1.4457 & -0.6672 & -0.5790 & 1.2516 & -0.8184 & -0.4790 \\
-1.3567 & -0.9348 & 0.7573 & 1.7002 & -0.9627 & -0.5655 & 2.5222 \\
1.6639 & 0.6111 & -0.1858 & 0.0485 & 0.1136 & 0.1372 & -0.0149 \\
-0.1400 & -0.3303 & -0.2965 & 0.0218 & 0.0565 & -0.1907 & -0.2544 \\
-1.2662 & 0.1905 & 0.3302 & -0.4041 & 1.1479 & -1.4716 & 0.0857
\end{array}\right)
$$

Given matrices $F$ and $G$ satisfy $\operatorname{rank}(G)=6$ and $G^{T} F \succeq 0$. According to the result given in [12], $F G^{+}$is the unique solution of the symmetric positive semidefinite Procrustes problem (1.1). Applying our algorithm, we also get

$$
\widehat{P}=\left(\begin{array}{rrrrrr}
5.4090 & 1.6334 & -0.3068 & 3.8723 & 5.4084 & 1.6336 \\
1.6334 & 9.6368 & -3.5302 & 2.4791 & 1.6334 & 9.6366 \\
-0.3068 & -3.5302 & 2.7113 & 0.0246 & -0.3069 & -3.5303 \\
3.8723 & 2.4791 & 0.0246 & 5.9698 & 3.8718 & 2.4793 \\
5.4084 & 1.6334 & -0.3069 & 3.8718 & 5.4077 & 1.6336 \\
1.6336 & 9.6366 & -3.5303 & 2.4793 & 1.6336 & 9.6367
\end{array}\right)=F G^{+}
$$

EXAmPle 3.2. Consider matrices $F$ and $G$ as follows:

$$
\begin{array}{r}
F=\left(\begin{array}{rrrrrrr}
3.7254 & 1.2702 & -4.7155 & 0.2991 & 3.7254 & 1.2702 & -1.1526 \\
0.1132 & 1.0888 & -0.8433 & 0.8514 & 0.1132 & 1.0888 & -0.6262 \\
1.9628 & 0.3865 & -3.7935 & 0.4054 & 1.9628 & 0.3865 & -0.6943 \\
-1.3531 & -0.4634 & 1.5233 & 0.6721 & -1.3531 & -0.4634 & -1.2785 \\
-0.6927 & -0.7689 & 1.0450 & -0.3898 & -0.6927 & -0.7689 & -1.2769 \\
-0.2666 & 4.2815 & -1.5041 & 2.0745 & -0.2666 & 4.2815 & 1.7293
\end{array}\right), \\
G=\left(\begin{array}{rrrrrrr}
-2.0558 & -0.4085 & -0.1904 & -0.6185 & -1.5556 & 0.6819 & 0 \\
0.3890 & -0.3175 & 0.2489 & -1.0140 & 0.0585 & -0.2079 & 0 \\
-0.5322 & 0.4753 & 1.5232 & -0.2299 & 0.4784 & 0.2653 & 0 \\
0.9646 & 1.0535 & -0.2607 & -1.7766 & -0.2345 & -0.5523 & 0 \\
0.0682 & -1.2079 & 0.0918 & 0.5055 & 0.5309 & 1.5097 & 0 \\
1.6319 & -2.6500 & 0.6705 & -1.0364 & 0.7542 & -0.7200 & 0
\end{array}\right) .
\end{array}
$$

Given matrices $F$ and $G$ satisfy $\operatorname{rank}(G)=6$ and $-F G^{T}-G F^{T} \succeq 0$. According to the result given in [12], the unique solution $\widehat{P}$ of the symmetric positive semidefinite Procrustes problem (1.1) is a zero matrix. Applying our algorithm, we also get $\widehat{P}=0$.

ExAmple 3.3. Consider matrices $F$ and $G$ as follows:

$$
F=\left(\begin{array}{rrrrrrr}
0.0591 & -5.4865 & -6.2530 & 0.0591 & -5.4865 & 5.0874 & 0 \\
-1.6696 & -2.3504 & 0.3833 & -1.6696 & -2.3504 & 2.1287 & 0 \\
-0.7507 & 0.9665 & 2.4886 & -0.7507 & 0.9665 & -1.1899 & 0 \\
-0.8736 & -0.7342 & 0.9214 & -0.8736 & -0.7342 & 0.4956 & 0 \\
0.2884 & 1.1065 & 0.5423 & 0.2884 & 1.1065 & 0.6941 & 0 \\
0 & 0 & 0 & 0 & 0 & 0 & 0
\end{array}\right)
$$




$$
G=\left(\begin{array}{rrrrrrr}
-0.0344 & -1.9744 & -1.8512 & -1.0034 & -1.3692 & 0 & 0.0736 \\
-0.7741 & -1.5501 & -0.9287 & -2.3712 & 0.9558 & 0 & 0.1784 \\
-0.0908 & -0.0294 & 0.5028 & -0.4758 & 0.7370 & 0 & 0.7718 \\
-0.5774 & -0.6471 & 0.4690 & 0.4695 & -0.1904 & 0 & -0.3603 \\
-0.7186 & 1.3223 & -0.4402 & -0.1210 & 0.0822 & 0 & 1.5890 \\
0 & 0 & 0 & 0 & 0 & 0 & 0
\end{array}\right) .
$$

Given matrices $F$ and $G$ satisfy $\operatorname{rank}(G)=5<6$ (this is show that $G$ is not a full row rank matrix) and

$$
\widehat{S}=\left(\begin{array}{rrrrr}
1.6938 & 0.9640 & -0.7144 & 0.2359 & 0.0315 \\
0.9640 & 0.9932 & -1.0090 & -0.0938 & 0.0169 \\
-0.7144 & -1.0090 & 1.2873 & 0.4194 & 0.0302 \\
0.2359 & -0.0938 & 0.4194 & 0.9759 & 0.1372 \\
0.0315 & 0.0169 & 0.0302 & 0.1372 & 0.0496
\end{array}\right) \succeq 0 .
$$

According to the result given in [7], $\widehat{P}_{11}$ in (2.24) should be equal to $\widehat{S}$. Applying our algorithm, we also get $\widehat{P}_{11}=\widehat{S}$, and hence, the solution of the symmetric positive semidefinite Procrustes problem (1.1) is

$$
\widehat{P}=\left(\begin{array}{rrrrrr}
2.7897 & -0.0361 & -0.9181 & 0.0287 & -0.2066 & 0 \\
-0.0361 & 0.5903 & 0.2470 & 0.3617 & -0.1641 & 0 \\
-0.9181 & 0.2470 & 0.5119 & 0.3893 & 0.0205 & 0 \\
0.0287 & 0.3617 & 0.3893 & 0.9896 & -0.0358 & 0 \\
-0.2066 & -0.1641 & 0.0205 & -0.0358 & 0.1183 & 0 \\
0 & 0 & 0 & 0 & 0 & 0
\end{array}\right) .
$$

EXAMPLE 3.4. Consider matrices $F$ and $G$ as follows:

$$
\begin{aligned}
F & =\left(\begin{array}{rrrrrrr}
2.4645 & 8.0375 & -0.3807 & 6.5545 & 6.2486 & -0.9846 & 0 \\
-6.3321 & -1.6502 & -3.1883 & -14.1036 & 0.5006 & 4.3716 & 0 \\
2.8313 & 5.4521 & -5.8879 & 2.9743 & 6.7764 & -1.5223 & 0 \\
3.6053 & -13.5812 & 3.9058 & 2.4470 & -8.4832 & -1.8689 & 0 \\
0.5485 & 1.8536 & 5.8800 & 1.3156 & 2.8428 & -2.0388 & 0 \\
0 & 0 & 0 & 0 & 0 & 0 & 0
\end{array}\right), \\
G & =\left(\begin{array}{rrrrrrr}
-0.1650 & 0.3558 & 1.0772 & -0.3787 & -0.0908 & 0 & 1.6613 \\
0.2620 & 0.6682 & -2.1780 & 0.3382 & -1.0792 & 0 & -0.4412 \\
-0.5204 & -0.1254 & 0.1140 & -0.5541 & -0.1034 & 0 & -0.4251 \\
0.6854 & -0.9204 & 0.1411 & 0.2553 & 0.4341 & 0 & 1.6297 \\
-0.5985 & 0.2386 & 0.3500 & 1.1481 & -0.2702 & 0 & 0.2986 \\
0 & 0 & 0 & 0 & 0 & 0 & 0
\end{array}\right) .
\end{aligned}
$$

Given matrices $F$ and $G$ satisfy $\operatorname{rank}(G)=3<6$ and $\widehat{S}$ is not a positive semidefinite symmetric matrix. Applying our algorithm, we get

$$
\widehat{P}_{11}=\left(\begin{array}{rrrrr}
1.5990 & 0.0438 & -0.5580 & -1.7145 & -0.2059 \\
0.0438 & 0.7856 & -1.4936 & 0.2236 & 0.6992 \\
-0.5580 & -1.4936 & 3.6000 & -0.7520 & -1.7244 \\
-1.7145 & 0.2236 & -0.7520 & 3.0715 & 1.0987 \\
-0.2059 & 0.6992 & -1.7244 & 1.0987 & 1.0133
\end{array}\right) \neq \widehat{S}
$$


and hence, the solution of the symmetric positive semidefinite Procrustes problem (1.1) is

$$
\widehat{P}=\left(\begin{array}{rrrrrr}
1.8432 & 0.2723 & -0.6009 & -2.2400 & 0.4686 & 0 \\
0.2723 & 1.7620 & 0.3597 & -0.7179 & -1.7996 & 0 \\
-0.6009 & 0.3597 & 0.3145 & 0.6743 & -0.6520 & 0 \\
-2.2400 & -0.7179 & 0.6743 & 3.9169 & -0.4570 & 0 \\
0.4686 & -1.7996 & -0.6520 & -0.4570 & 2.2329 & 0 \\
0 & 0 & 0 & 0 & 0 & 0
\end{array}\right) .
$$

Acknowledgements. The authors thank an anonymous referee for valuable suggestions which helped them to improve this article.

\section{REFERENCES}

[1] B. Adhikari and R. Alam. Structured Procrustes problem. Linear Algebra Appl., 490:145-161, 2016.

[2] A. Albert. Condition for positive and nonnegative definite in terms of pseudoinverse. SIAM J. Appl. Math., 17:434-440, 1969.

[3] J.C. Allwright. Positive semidefinite matrices: Characterization via conical hulls and least-squares solution of a matrix equation. SIAM J. Control Optim., 26:537-556, 1988.

[4] J.E. Brock. Optimal matrices describing linear systems. AIAA J., 6:1292-1296, 1968.

[5] N. Gillis and P. Sharma. A semi-analytical approach for the positive semidefinite procrustes problem. Linear Algebra Appl., 540:112-137, 2018.

[6] R.A. Horn and C.R. Johnson. Topics in Matrix Analysis. Cambridge University Press, Cambridge, 1991.

[7] A.P. Liao. On the least squares problem of a matrix equation. J. Comput. Math., 17:589-594, 1999.

[8] T. Suffridge and T. Hayden. Approximation by a Hermitian positive semidefinite Toeplitz matrix. SIAM J. Matrix Anal. Appl., 14:721-734, 1993.

[9] J.G. Sun. Least squares solution of a calss of inverse eigenvalue problems. Math. Numer. Sin., 2:206-216, 1987.

[10] J.G. Sun. Two kinds of inverse eigenvalue problems for real symmetric matrices. Math. Numer. Sin., 3:282-290, 1988.

[11] K.G. Woodgate. A new algorithm for the positive semi-definite Procrustes problem. Proceedings of the 32nd Conference on Decision \& Control, San Antonio, 3596-3601, 1993.

[12] K.G. Woodgate. Least-squares solution of $F=P G$ over positive semidefinite symmetric P. Linear Algebra Appl., 245:171-190, 1996.

[13] L. Zhang. A class of inverse eigenvalue problem for symmetric nonnegative definite matrices. J. Hunan Educational Inst., 2:11-17, 1995 (in Chinese). 